\title{
Mineralization of Decalcified Bone Occurs Under Cell Culture Conditions and Requires Bovine Serum But Not Cells
}

\author{
N. J. Hamlin, P. A. Price \\ Division of Biological Sciences, University of California, San Diego, La Jolla, CA 92093-0368, USA
}

Received: 21 June 2003 / Accepted: 9 February 2004 / Online publication: 28 May 2004

\begin{abstract}
The purpose of this study was to develop an in vitro model system for bone matrix mineralization in the absence of cells. For this model, we utilized EDTAdecalcified new-born rat tibias with the cartilaginous ends intact, allowing us to visually determine the specificity of mineralization within the bone. Our results show that supplementation of DMEM culture medium with $10 \mathrm{mM} \beta$-glycerophosphate and $15 \%$ fetal bovine serum (FBS) results in non-physiological mineral percipitation in the tibia because of the generation of supraphysiological $(5 \mathrm{mM})$ levels of inorganic phosphate in the medium. The same medium supplemented only with inorganic phosphate to a final concentration of $2 \mathrm{mM}$ failed to mineralize a decalcified tibia matrix. However, additional supplementation of this medium with as little as $5 \%$ FBS resulted in mineralization of those regions of the type I collagen where mineral was found prior to decalcification, with no evidence for mineralization in the cartilage at the bone ends or in the periosteum. Analysis of the mineral by Fourier-transform infrared spectroscopy and powder X-ray diffraction shows that tibias that have been decalcified and then remineralized contain an apatitic mineral that is strikingly similar to the mineral in normal bone. Tendon, a type I collagen matrix not normally mineralized in vivo, also mineralizes when incubated in DMEM containing $2 \mathrm{mM} \mathrm{Pi}$ and as little as $1.5 \%$ FBS, but not when incubated in DMEM without serum. These data indicate that serum contains a nucleator of type I collagen matrix mineralization, and that mineralization of type I collagen under cell culture conditions requires serum but not living cells.
\end{abstract}

Key words: Calcification of bone - Calcification of tendon - Serum nucleator — Type I collagen

Cell culture models have been used extensively to study matrix mineralization in vitro. The majority of these studies have utilized an osteoblastic cell line in culture media that typically contain ionic calcium levels between $1.8 \mathrm{mM}$ [Minimum Essential Medium (MEM), aMEM, and Dulbecco's Modified Eagle Medium (DMEM)] and $2.6 \mathrm{mM}$ [Biggers, Gwatkin and Judah b (BGJ/b) Medium, Fitton Jackson Modification], ionic phosphate levels of $0.9 \mathrm{mM}$ (DMEM) to $1.6 \mathrm{mM}(\mathrm{BGJ} / \mathrm{b})$, and $10 \mathrm{mM} \beta$-glycerophosphate [1-14]. The role of the $\beta$ glycerophosphate is generally accepted to be as a source of phosphate for mineral growth, but it may affect mineral deposition in other ways as well [15]. Alkaline phosphatase secreted by bone cells efficiently hydrolyzes the added $10 \mathrm{mM} \beta$-glycerophosphate $[16,17]$ and increases medium phosphate in osteoblast cultures to $7-$ $9 \mathrm{mM}$ within 24 hours [17]. Khouja et al. [14] have shown that serum, in the absence of cells, is also able to hydrolyze $\beta$-glycerophosphate, presumably because of endogenous action of serum alkaline phosphatase.

One of the goals of our laboratory is to develop a simple, reproducible, cell-free model to study the initiation and inhibition of bone matrix mineralization in vitro. We report here on the remineralization of demineralized bone in cell culture medium containing fetal bovine serum. Demineralized newborn rat tibias were used as a model bone matrix in these studies so that the location of any in vitro mineralization observed could be compared directly with the location of mineral found in the original tibia prior to demineralization.

\section{Materials and Methods}

\section{Materials}

Forty-day-old male rats (Sprague-Dawley-derived) were purchased from Charles River Labs (Wilmington, MD) and newborn rats were generously provided by Dr. Kenneth Chien (UCSD). Dulbecco's Modified Eagle Medium (DMEM), penicillin-streptomycin, and fetal bovine serum (FBS) were purchased from Gibco (Grand Island, NY), $\beta$-glycerophosphate and Alizarin red S were purchased from Sigma (St. Louis, MO), and sodium azide was purchased from EM Science (Gibbstown, NJ). DMEM was supplemented with penicillin-streptomycin and $0.02 \%$ sodium azide to prevent bacterial growth. Where indicated, the phosphate concentration of DMEM was increased from the basal $0.9 \mathrm{mM}$ to a final $2 \mathrm{mM}$ by the addition of $0.5 \mathrm{M}$ sodium phosphate buffer $\mathrm{pH} 7.4$, using vigorous stirring to achieve rapid dispersal and prevent precipitation of a calcium phosphate mineral phase. Phosphate-boosted DMEM could be stored at $4^{\circ} \mathrm{C}$ for up to 6 weeks without evidence for the formation of a calcium phos- 
phate mineral phase. All chemicals employed were reagent grade or better.

\section{Preparation of Tibia and Tendon}

Rats were killed while under isoflurane anesthetic by exsanguination (40-day-old) or by decapitation (newborn). Tibias were dissected from newborn rats, demineralized in $0.5 \mathrm{M}$ ethylenediaminetetraacetate (EDTA) $(\mathrm{pH} 7.5)$ for between 24 and 72 hours at room temperature, washed exhaustively with ultrapure water to remove all traces of EDTA, and stored at $-20^{\circ} \mathrm{C}$ until use. Tendons were obtained from the tails of $40-$ day-old rats and extracted with EDTA as described above and dried. The UCSD Animal Subjects Committee approved all animal experiments.

\section{Calcification Procedures}

All experiments were performed under aseptic conditions using a humidified incubator at $37^{\circ} \mathrm{C}$ and $5 \% \mathrm{CO}_{2}$. The calcification of demineralized rat tibias and tendons was tested in $100-\mathrm{mm}$ culture dishes (Falcon 3803) containing $10 \mathrm{ml}$ of DMEM (containing 0.9 or boosted to $2 \mathrm{mM} \mathrm{Pi}$ ), fetal bovine serum, or mixtures of the two. After equilibration with $5 \% \mathrm{CO}_{2}$ at $37^{\circ} \mathrm{C}$, all incubation media maintained a stable $\mathrm{pH}$ value of 7.4 throughout the duration of the 6-day incubation. The medium was not changed during the 6-day incubation. Dry tendon segments were rehydrated by overnight equilibration in ultrapure water before use. A single demineralized tibia or a hydrated tendon sample ( $3 \mathrm{mg}$ initial dry weight) was added to each $100 \mathrm{~mm}$ plate.

For the $\beta$-glycerophosphate time course experiment, $21.6 \mathrm{mg}$ of $\beta$-glycerophosphate was added to $10 \mathrm{ml}$ culture medium to give a final concentration of $10 \mathrm{mM}$. Samples were incubated for 6 days at $37^{\circ} \mathrm{C}, 5 \% \mathrm{CO}_{2}$, with $300-\mu \mathrm{l}$ aliquots of medium taken at $t=0$ and every 24 hours thereafter. Aliquots were placed in 1.5-ml eppifuge tubes, frozen on dry ice, and stored at $-20^{\circ} \mathrm{C}$ for later phosphate determination.

\section{Biochemical Analyses}

For assessment of calcification, each tibia or tendon was removed from the incubation solution, placed in a $15-\mathrm{ml}$ conical tube containing $10 \mathrm{ml}$ of $0.5 \% \mathrm{KOH}$ (weight \%) with $0.002 \%$ Alizarin red S, and mixed end-over-end for 24 hours. The stain solution was then replaced with $0.05 \% \mathrm{KOH}$, mixed for another 24 hours, and the tissue was photographed; stained tissues were stored in glycerin. For histological analyses, tibias and tendons were fixed for 1 day at room temperature in absolute ethanol and sent to San Diego Pathology Inc. (San Diego, CA) for embedding, sectioning, and von Kossa staining. For quantitative assessment of the extent of calcification, tibias and tendons were extracted for 24 hours at room temperature with $2 \mathrm{ml}$ of $0.15 \mathrm{M} \mathrm{HC1}$. Calcium levels in the acid extracts were determined colorimetrically using cresolphthalein complexone (Sigma) and phosphate levels were determined colorimetrically as described [18].

\section{Alkaline Phosphatase Activity in Fetal Bovine Serum}

Alkaline phosphatase activity was determined using an alkaline phosphatase liquid reagent kit, a kinetic colorimetric assay using $p$-nitrophenylphosphate as substrate, and carried out as described by the manufacturer (JAS Diagnostics, Inc., Miami, FL).

\section{Fourier Transform Infrared (FTIR) Spectroscopy}

Ten newborn rat tibias were dissected, the cartilaginous ends removed, and lyophilized to dryness. Demineralized tibias, remineralized in $15 \%$ FBS as described above, were removed

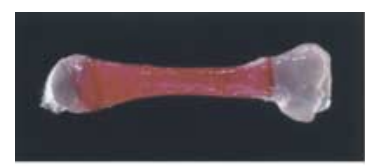

Newborn Rat Tibia

Demineralized tibia incubated 6 days in DMEM with:

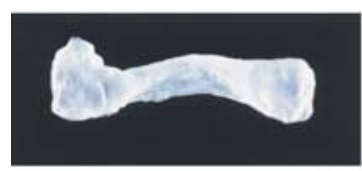

$15 \%$ FBS

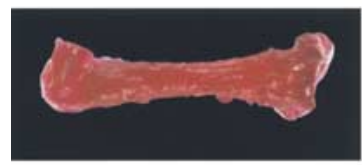

$15 \% \mathrm{FBS}+$

$10 \mathrm{mM} \beta$-glycerophosphate

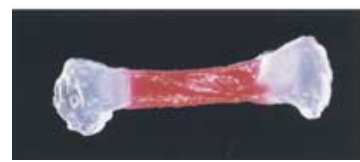

$15 \%$ FBS + Phosphate to $2 \mathrm{mM}$

Fig. 1. Calcification of demineralized rat tibias after incubation for 6 days DMEM containing 15\% FBS and added $\beta$ glycerophosphate or Pi. Newborn rat tibias were demineralized for 72 hours with $0.5 \mathrm{M}$ EDTA ( $\mathrm{pH} 7.5$ ) and rinsed thoroughly with water. Demineralized tibias were placed in $100-\mathrm{mm}$ plastic tissue culture dishes containing $10 \mathrm{ml}$ DMEM and $15 \%$ FBS (v/v) either alone, with $10 \mathrm{mM} \beta$-glycerophosphate, or with enough added phosphate to make the final concentration $2 \mathrm{mM}$. A control tibia is shown before demineralization and after Alizarin red staining. Demineralized tibias were incubated for 6 days at $37^{\circ} \mathrm{C}$ and $5 \% \mathrm{CO}_{2}$ and then placed into Alizarin red stain. A representative tibia from each incubation condition is shown. The Alizarin red staining indicates sites of calcification (see online figure for a color image).

from their medium after 6 days, the cartilaginous ends removed, and lyophilized to dryness. Dried tibias were ground with an agate mortar and pestle. Ground bone was mixed with FTIR-grade potassium bromide (Aldrich Chemical Company, Milwaukee, WI) in a ratio of approximately $3 \mathrm{mg}$ ground bone to $100 \mathrm{mg} \mathrm{KBr}$. A portion of this material was pressed into a transparent pellet using a minipellet maker. The pellet was analyzed on a Nicolet Series II Magna-IR 550 Spectrometer from 2000 to $400 \mathrm{~cm}^{-1}$ at $4-\mathrm{cm}^{-1}$ resolution for 256 scans. The data were collected using Omnic QuickIR software in the Basic FTIR mode set to measure absorbance.

Normal tibia mineral was compared with mineral present in tendon mineralized in 15\% FBS in DMEM for 6 days as follows. Ten 3-mg portions of dried rat tail tendon were hydrated, then incubated in DMEM containing 15\% FBS for 6 days as described above. To better compare the mineral phases in normal tibias and mineralized tendon, samples were then deproteinized as described [19] and compared with deproteinized newborn rat tibias. Deproteinized mineral from the tendon and tibias were each combined with $100 \mathrm{mg} \mathrm{KBr}$, pressed into a pellet, and analyzed exactly as was done with the nondeproteinized tibia samples described above.

\section{Powder X-Ray Diffraction}

Ground bone samples and deproteinized bone and tendon samples were prepared as above for FTIR analysis up 


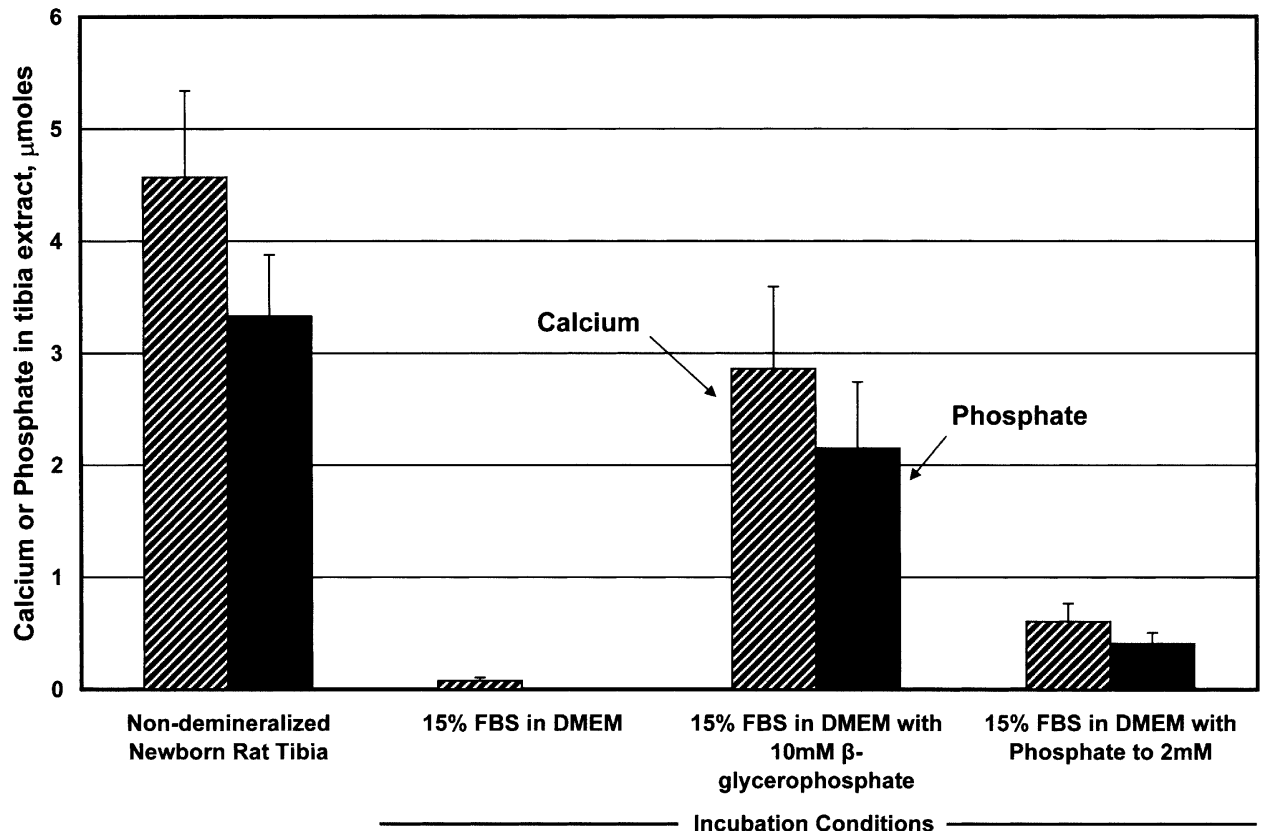

Fig. 2. Calcium and phosphate levels in tibias after incubation for 6 days in DMEM containing 15\% FBS and added $\beta$-glycerophosphate or Pi. Demineralized newborn rat tibias were incubated as described in the Figure 1. After incubation, tibias were demineralized in acid and the acid extracts were analyzed for calcium and phosphate as described in Materials and Methods. Nondemineralized control tibias were also acid extracted and analyzed for calcium and phosphate. Error bar represents one standard deviation above the mean, $N=4$ for each condition.

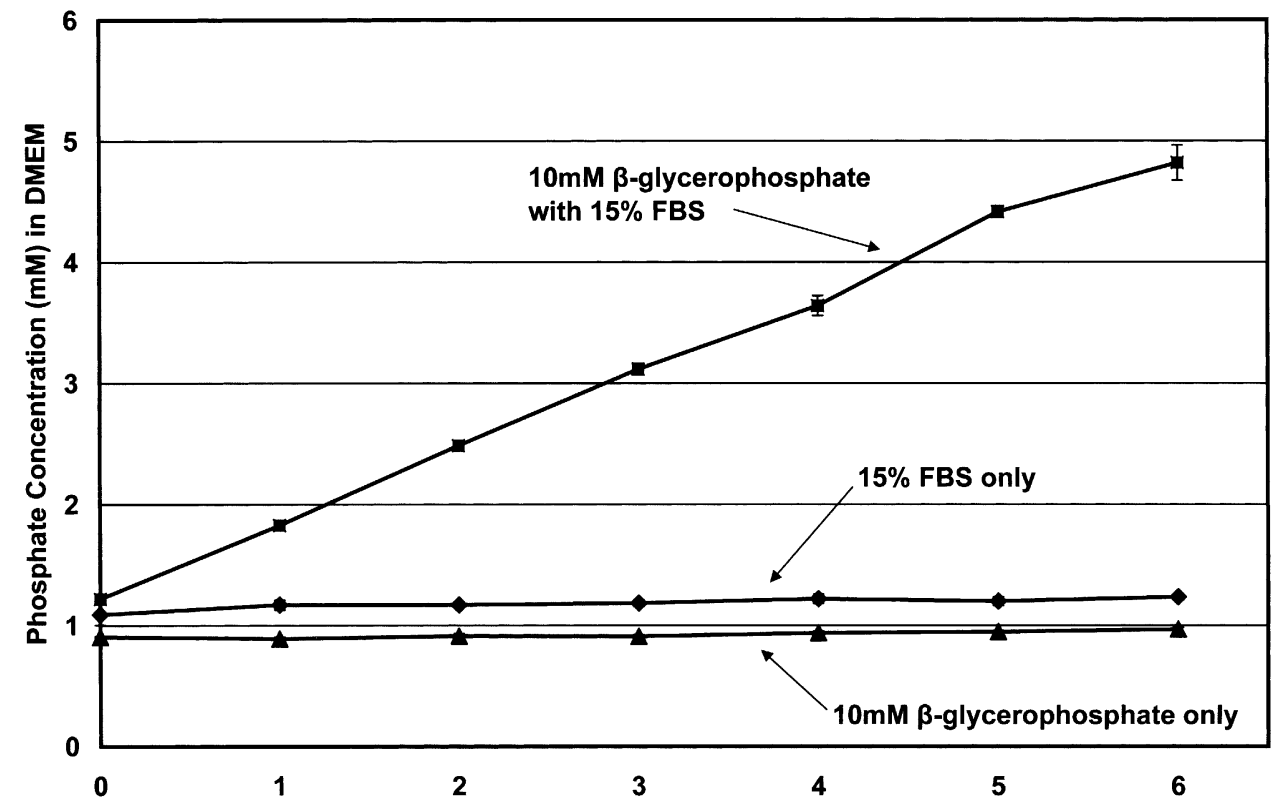

Fig. 3. Phosphate levels increase in DMEM containing $10 \mathrm{mM} \beta$-glycerophosphate and $15 \%$ FBS. Ten milliliters of DMEM containing $15 \%$ FBS (v/v) were added to two $100-\mathrm{mm}$ tissue culture plates and $10 \mathrm{ml}$ DMEM without FBS were added to a third. One of the plates containing serum and the plate without serum received enough $\beta$-glycerophosphate to bring the initial concentration to $10 \mathrm{mM}$. All three plates were placed in a humidified incubator for 6 days at $37^{\circ} \mathrm{C}$ and $5 \%$
$\mathrm{CO}_{2}$. Aliquots of media were taken every 24 hours and assayed for phosphate (see Materials and Methods). Each experimental condition was replicated 4 times. The graph shows the level of phosphate in DMEM containing $10 \mathrm{mM} \beta$-glycerophosphate and $15 \%$ FBS $(\bigcirc), 15 \%$ FBS only $(\diamond)$, and $10 \mathrm{mM} \beta$-glycerophosphate only $(\boldsymbol{\Delta})$. Error bars indicate one standard deviation above and below the mean, $N=4$ for each condition. Some error bars are not visible but are present. through the step before adding $\mathrm{KBr}$. Ten to 20 tibias or 10 20 3-mg tendon portions, enough to obtain sufficient powder sample, were analyzed on a Scintag SDF 2000 X-ray diffractometer. Samples were scanned from 1-70 deg at a normal, continuous scan rate of $2.0 \mathrm{deg} /$ minute and a step size of $0.02 \mathrm{deg}$.

\section{Statistical Analyses}

All medium phosphate, mineral extract, and $\mathrm{Ca} / \mathrm{PO}_{4}$ ratio data are presented as the mean $\pm \mathrm{SD}$. Statistical significance between groups was determined using the Student's $t$-test. A $P<$ 0.05 was considered significant. All sample sizes were $N=4$. 


\section{Results}

Calcification of Demineralized Rat Tibia by Incubation in $D M E M$ Containing FBS and added $\beta$-glycerophosphate

Most published in vitro cell mineralization studies employ osteoblastic cells in a standard cell culture medium, such as DMEM containing FBS, and supplement this medium with $\beta$-glycerophosphate. Our first experiments were carried out to determine whether demineralized bone would calcify when incubated for 6 days at $37^{\circ} \mathrm{C}$ in this culture medium in the absence of living cells. Newborn rat tibias were first freed of endogenous mineral by extraction for 72 hours with $0.5 \mathrm{M}$ EDTA ( $\mathrm{pH}$ 7.5), a procedure that also killed all cells originally found in the bone. As shown in Figures 1 and 2, there was calcification in the tibia after incubation in DMEM with $15 \%$ FBS plus $10 \mathrm{mM} \beta$-glycerophosphate, but there was no evidence for calcification in tibias incubated in DMEM with 15\% FBS alone. However, the calcification with $\beta$-glycerophosphate occurred in the cartilage at the ends of the tibia, as well as in bone matrix, and is an indication of nonphysiological mineral precipitation rather than specific mineralization. The molar ratio of calcium to phosphate in tibias remineralized in the presence of $\beta$-glycerophosphate, $1.34 \pm 0.04$, is not significantly different from that in newborn rat tibias, $1.37 \pm 0.03(P=0.25)$.

Previous studies have shown that over $70 \%$ of the $\beta$ glycerophosphate added to osteoblastic cell cultures is hydrolyzed to phosphate within 24 hours by cell-associated alkaline phosphatase [17], and that the accompanying massive increase in medium phosphate results in the nonspecific deposition of calcium phosphate mineral in the matrix [14-17]. Since there was clearly nonspecific deposition of mineral in tibias incubated in medium containing $10 \mathrm{mM} \beta$-glycerophosphate (particularly in the cartilaginous ends), it seemed likely that the hydrolysis of $\beta$-glycerophosphate to phosphate also occurred in the present studies as a result of endogenous alkaline phosphatase activity in the FBS. This possibility is supported by the observation that $\beta$-glycerophosphate added to human plasma is indeed efficiently hydrolyzed to phosphate [14].

To test this possibility, DMEM containing $10 \mathrm{mM} \beta$ glycerophosphate alone, $15 \%$ FBS alone, and both were incubated for 6 days in the absence of tibias. As seen in Figure 3, there was a linear increase from 0.9 to $4.82 \mathrm{mM}$ phosphate over the 6-day incubation in DMEM containing $10 \mathrm{mM} \beta$-glycerophosphate and $15 \%$ FBS, but there was no increase in phosphate in DMEM containing $10 \mathrm{mM} \beta$-glycerophosphate alone or $15 \%$ FBS alone. These results confirm that serum alkaline phosphatase activity, which we measured to be 0.24 $\mathrm{U} / \mathrm{ml}$ in FBS, can hydrolyze $\beta$-glycerophosphate to phosphate under conditions typically used in cell culture

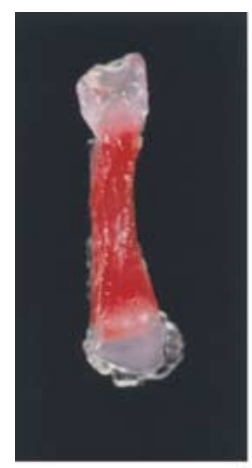

$100 \%$ FBS

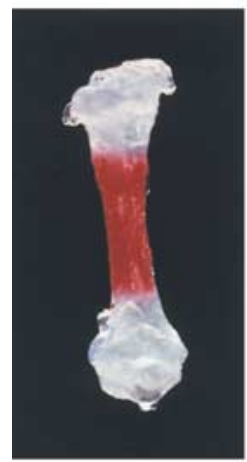

$1.5 \%$ FBS

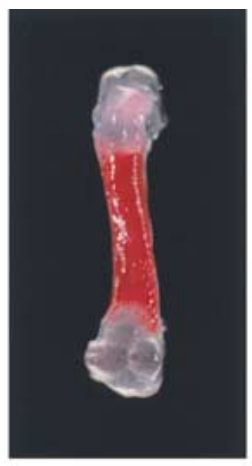

$15 \%$ FBS

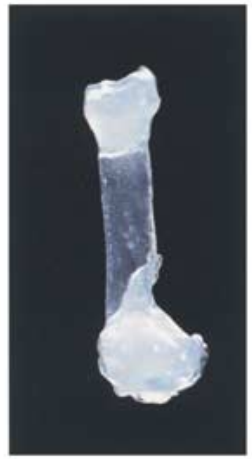

$0.15 \%$ FBS

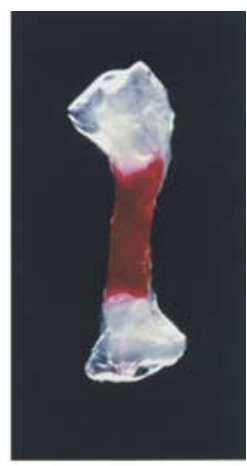

$5 \%$ FBS

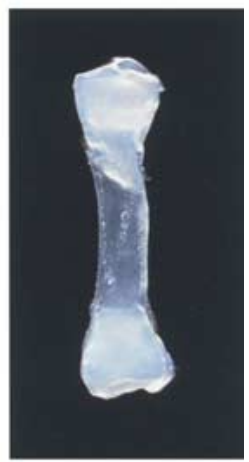

DMEM only

Fig. 4. Calcification of demineralized rat tibias after incubation in DMEM containing $2 \mathrm{mM}$ phosphate and the indicated amounts of FBS or in FBS alone (100\%). Demineralized newborn rat tibias were placed in $10 \mathrm{ml}$ DMEM containing $2 \mathrm{mM}$ phosphate and the indicated amounts of FBS or in $10 \mathrm{ml} \mathrm{FBS}$ alone and were incubated for 6 days in a humidified incubator at $37^{\circ} \mathrm{C}$ and $5 \% \mathrm{CO}_{2}$. After incubation, tibias were stained for calcification with Alizarin red (see online figure for color image). Shown is a representative tibia from each condition.

mineralization studies. This hydrolysis, however, results in a decidedly nonphysiological final ion product $\left(1.8 \mathrm{mM}[\mathrm{Ca}] \times 4.8 \mathrm{mM}[\mathrm{Pi}]=8.6 \mathrm{mM}^{2}\right.$ vs. $4 \mathrm{mM}^{2}$ for rat serum), an ion product that, when achieved by the direct addition of phosphate to DMEM, results in the massive precipitation of a calcium phosphate mineral phase within 1 day (personal observations). We conclude that the nonspecific calcification seen here in a tibia incubated in medium containing $10 \mathrm{mM} \beta$-glycerophosphate is due to this high calcium phosphate ion product.

Calcification of Demineralized Rat Tibia by Incubation in DMEM Containing FBS and Added Pi to a Final $2 \mathrm{mM}$ Concentration

We were unsatisfied with a model in which mineralization may be largely due to nonphysiological mineral precipitation. Using the approach of Chung et al. [17], we decided to boost medium phosphate to $2 \mathrm{mM}$ so that we could more directly control medium phosphate lev- 


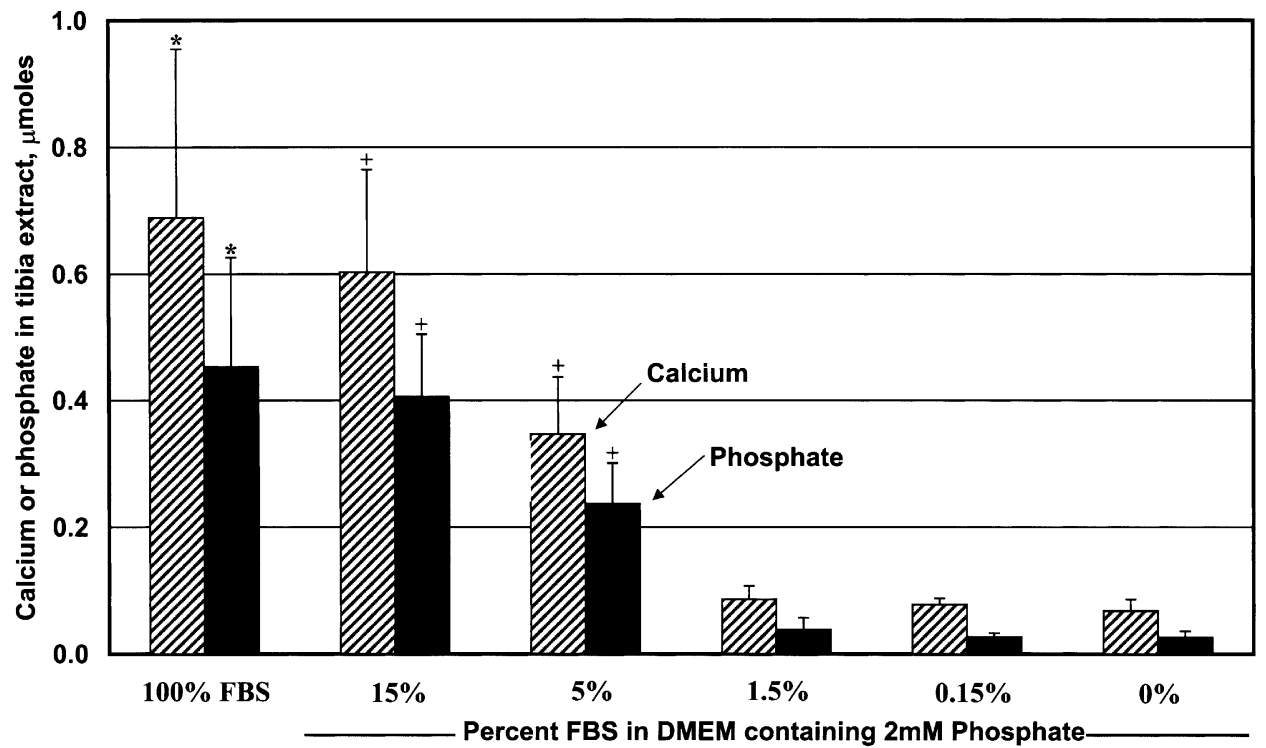

Fig. 5. Calcium and phosphate levels in tibias after incubation for 6 days in DMEM containing $2 \mathrm{mM}$ phosphate and the indicated amounts of FBS or in FBS alone. Demineralized newborn rat tibias were incubated as described in Figure 4. After incubation, tibias were demineralized in acid and the acid extracts were analyzed for calcium and phosphate as de-

els. This level of ionic phosphate, along with the $1.8 \mathrm{mM}$ $\mathrm{Ca}^{2+}$ present in DMEM, would give an ion product of $1.8 \mathrm{mM}[\mathrm{Ca}] \times 2 \mathrm{mM}[\mathrm{Pi}]=3.6 \mathrm{mM}^{2}$, a value that is much closer to that found normally in rat serum, $1.3 \mathrm{mM}[\mathrm{Ca}] \times 3.1 \mathrm{mM}[\mathrm{Pi}]=4.0 \mathrm{mM}^{2}$. Figures 1 and 2 show that mineralization did occur in 6 days, simply in medium supplemented with Pi to $2 \mathrm{mM}$ and $15 \%$ FBS (ion product $=3.7 \mathrm{mM}^{2}$ ). The pattern of Alizarin red staining for calcification in the tibia incubated in media with $2 \mathrm{mM}$ phosphate closely resembled the pattern of staining seen in the newborn rat tibia prior to demineralization, with intense staining for calcification in the bone of the midshaft region and absence of staining in the cartilaginous tissue at either end of the tibia (Fig. 1). While the absolute amount of mineral that was placed back into the matrix was not as great as what was initially present in the tibia (Fig. 2), the pattern of mineralization was more physiologically correct than the massive and nonspecific precipitation of mineral obtained throughout the tibias incubated in the presence of $\beta$-glycerophosphate. The ratio of calcium to phosphate in the remineralized tibias was $1.48 \pm 0.04$, which is higher than the $1.37 \pm 0.03$ ratio found in normal tibias $(P<0.01)$.

\section{Calcification of Demineralized Rat Tibia by Incubation in} DMEM Containing Different Amounts of FBS or in FBS Alone

The next experiments examined the role of the FBS component of the cell culture medium in the calcification of demineralized tibias. Because we no longer used scribed in Materials and Methods. Each condition was reproduced 4 times. The error bars represent one standard deviation above the mean. Values indicated $(*)$ are significantly different from $0 \%$ FBS $(P<0.01)$. Values indicated $(+)$ are significantly different from $0 \%$ FBS $(P<0.001)$.

$\beta$-glycerophosphate as a substrate for increasing medium phosphate, we suspected that FBS might be unnecessary as well. After all, in the absence of cells, FBS was not needed to provide growth factors and other nutrients, and serum alkaline phosphatase was not needed to generate phosphate from $\beta$-glycerophosphate. We suspected that simply boosting DMEM [Pi] to $2 \mathrm{mM}$ would be sufficient for mineralization to occur. Demineralized rat tibias were incubated for 6 days in DMEM containing $2 \mathrm{mM}$ phosphate alone or with $15 \%$ (calcium phosphate ion product $=3.7 \mathrm{mM}^{2}$ ), $5 \%$ $\left(3.6 \mathrm{mM}^{2}\right), 1.5 \%\left(3.6 \mathrm{mM}^{2}\right)$, or $0.15 \%\left(3.6 \mathrm{mM}^{2}\right)$ FBS. Surprisingly, as can be seen in Figure 4, tibias incubated in $15 \%, 5 \%$, and $1.5 \%$ FBS stained intensely for calcification with Alizarin red, while tibias incubated in $0.15 \%$ FBS or in serum-free medium did not stain. ${ }^{1}$ These findings indicated that something in serum is required in order to achieve calcification, and so we next incubated a tibia in $100 \%$ FBS $(1.3 \mathrm{mM}[\mathrm{Ca}] \times 3.1 \mathrm{mM}$ $\left[\mathrm{PO}_{4}\right]=4.0 \mathrm{mM}^{2}$ ). Figure 4 shows a representative tibia incubated for 6 days in FBS alone also calcified. The pattern of Alizarin red staining for calcification in tibias

\footnotetext{
${ }^{1}$ The failure of demineralized tibias to remineralize during incubation in serum-free DMEM clearly shows that serum-initiated remineralization is not due to the possible presence of calcium phosphate crystals that remained in the bone matrix after demineralization for 72 hours with $0.5 \mathrm{M}$ EDTA. This conclusion is further supported by the observation that tibias that have been demineralized in $150 \mathrm{mM} \mathrm{HCl}$ for 24 hours also remineralize in DMEM, but only if serum is present (personal observations). The serum-free DMEM used in the present study did, however, sustain the growth of added hydroxyapatite
} crystals (data not shown). 
incubated in $100 \%$ FBS or in DMEM containing $15 \%$, $5 \%$, or $1.5 \%$ FBS again matched the pattern of staining seen in the newborn rat tibia prior to demineralization (Fig. 1), with intense staining for calcification in the bone of the midshaft region and absence of staining in the cartilaginous tissue at either end of the tibia. These experiments have been repeated over 30 times in an 18month period, and in every experiment demineralized tibias incubated for 6 days in DMEM containing $15 \%$, $5 \%$, or $1.5 \%$ FBS stained consistently with Alizarin red, while tibias incubated in DMEM alone did not stain.

Additional experiments were carried out to further characterize the calcification induced by incubation of demineralized tibias for 6d in FBS alone, or in DMEM containing different amounts of FBS. As seen in Figure 5 , chemical analyses of tibia extracts confirmed most of the Alizarin staining results and showed a dramatic accumulation of calcium and phosphate in tibias incubated for 6 days in FBS alone or in DMEM containing $15 \%$ or $5 \%$ FBS. The ratios of calcium to phosphate extracted from tibias incubated in FBS alone $(1.52 \pm 0.02), 15 \%$ FBS $(1.48 \pm 0.04)$, and $5 \%$ FBS $(1.47 \pm 0.02)$ are all higher than that from nondemineralized tibias $(1.37 \pm 0.03, P<0.01)$. Incubation in $1.5 \%$ FBS typically results in a positively Alizarin stained tibia, but mineral extracts often show low levels of calcium and phosphate uptake. No evidence for accumulation of calcium and phosphate was seen in tibias incubated in $0.15 \%$ FBS or in DMEM alone. The microscopic pattern of calcification induced by incubating demineralized tibias in FBS alone or in DMEM containing $15 \%$ or $1.5 \%$ FBS is nearly identical to that seen in tibias prior to demineralization, with intense von Kossa staining for phosphate mineral in the type I collagen matrix of bone and no evidence for calcification in the periosteum (Fig. 6) or in the cartilaginous tissue at the bone ends (not shown). The calcification induced by incubation of demineralized tibias in FBS or in DMEM containing $15 \%$ or $1.5 \%$ FBS is discontinuous, however, with numerous discrete calcification foci, while calcification in the newborn rat tibia prior to demineralization is uniform and continuous.

We employed FTIR spectroscopy and powder X-ray diffraction to compare the mineral phase found in the remineralized tibia to that present in the original tibia prior to demineralization. As seen in Figure 7, the FTIR absorbance traces of nondemineralized newborn rat tibias and remineralized tibias are strikingly similar. Both spectra show the presence of phosphate, with a $\mathrm{P}-\mathrm{O}$ stretch vibration absorbance peak at around $1030 \mathrm{~cm}^{-1}$, and $\mathrm{P}-\mathrm{O}$ bend vibration absorbance peaks at around 600 and $560 \mathrm{~cm}^{-1}$. Additionally, each spectrum has a carbonate $\mathrm{C}-\mathrm{O}$ bend vibration absorbance at around $872 \mathrm{~cm}^{-1}$ and a $\mathrm{C}-\mathrm{O}$ stretch vibration absorbance at around $1400-1460 \mathrm{~cm}^{-1}$ [20]. Powder Xray diffraction analysis shows that nondemineralized

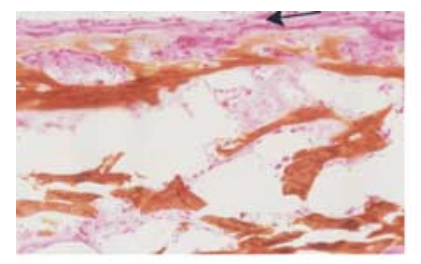

Non-Demineralized Tibia

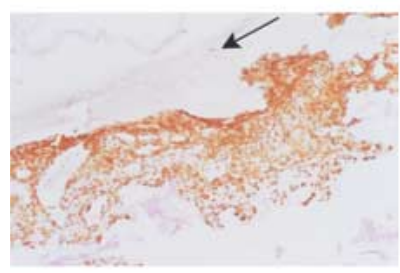

$15 \%$ FBS

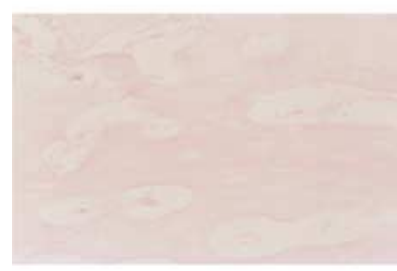

$0.15 \%$ FBS

Fig. 6. Microscopic examination of tibias for mineral after incubation for 6 days in DMEM containing $2 \mathrm{mM}$ phosphate and the indicated amounts of FBS or in FBS alone. Demineralized newborn rat tibias were incubated as described in Figure 4 and then fixed in absolute ethanol. Representative von Kossa-stained sections are shown for an original tibia before demineralization (upper left) and for demineralized tibias after incubation for 6 days at $37^{\circ} \mathrm{C}$ in DMEM containing $2 \mathrm{mM}$ phosphate and the indicated amounts of FBS or in FBS alone (upper right). von Kossa staining indicates the presence of phosphate mineral. The arrows indicate the location of the periosteum, if present in a section. (See online figure for a color image). $400 \times$ magnification.

tibias and remineralized tibias both contain a major diffraction peak at $32 \mathrm{deg}$ (Figure 7 insets), a characteristic of bone apatite.

Calcification of Rat Tail Tendon by Incubation in FBS or in DMEM Containing Different Amounts of FBS

The above experiments indicate that the calcification of demineralized tibias in DMEM containing FBS is restricted to those matrix regions that were calcified in the original bone. This calcification pattern suggests that the calcification that occurs under these conditions may be specific for type I collagen, which is the extracellular matrix found in bone as well as in tendon and other connective tissues that do not normally calcify. We wondered whether other type I collagen matrices would calcify under the conditions of our model system and, to test this possibility, the tail tendons from 40 -day-old rats 

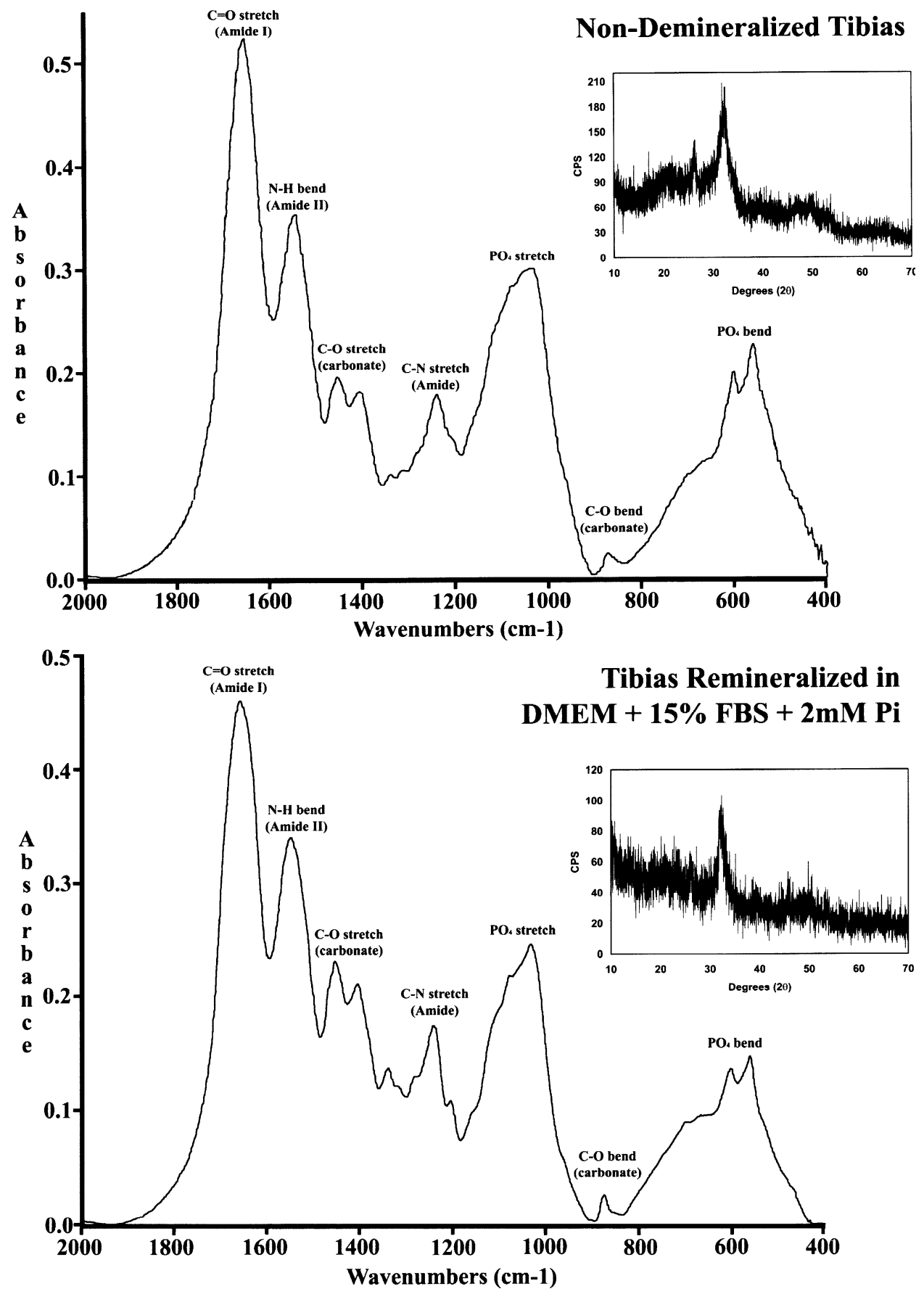

Fig. 7. Fourier transform infrared (FTIR) spectroscopic and powder X-ray diffraction (XRD) analyses of normal and remineralized tibias. Tibias were demineralized and remineralized in $15 \% \mathrm{FBS}$, and normal and remineralized tibias were prepared for FTIR analysis as described in Materials and Methods. $\mathrm{KBr}$ transparent pellets were analyzed from 2000 to $400 \mathrm{~cm}^{-1}$ at $4-\mathrm{cm}^{-1}$ resolution for 256 scans, (Top) Newborn rat tibias. (Bottom)

Remineralized newborn rat tibias. Ground tibias were analyzed on a Scintag SDF $2000 \mathrm{X}$-ray diffractometer as described in Materials and Methods (insets). were first extracted for 24 hours with $0.5 \mathrm{M}$ EDTA $(\mathrm{pH} 7.5)$ and then incubated for 6 days in DMEM containing $2 \mathrm{mM}$ phosphate and different amounts of FBS. As seen in Figure 8, tendons incubated for 6 days in FBS or in DMEM containing $15 \%$ or $1.5 \%$ FBS stained intensely with Alizarin red, while tendons incubated in $0.15 \%$ FBS or in serum-free DMEM did not stain. Chemical analyses of tendon extracts (Fig. 9) showed that tendons incubated for 6 days in FBS or in DMEM containing $15 \%$ or $1.5 \%$ FBS also had increased levels of calcium and phosphate when compared to tendons incubated in DMEM alone. These effects of FBS on the accumulation of calcium and phosphate in tendons are similar to those seen with demineralized tibias (Fig. 5). However, the overall amount of calcium phosphate mineral deposited into tendon was about tenfold higher than for tibia. This result may be due to the greater amount of collagen matrix in the 3-mg tendon samples than is present in the newborn tibia, although this remains to be proven. Additionally, the $\mathrm{Ca} /$ $\mathrm{PO}_{4}$ ratios determined from mineral extracts of tendon (Fig. 9) after incubation in $100 \%$ FBS $(1.43 \pm 0.08)$, 


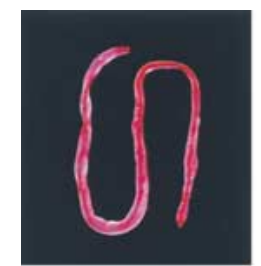

$100 \%$ FBS

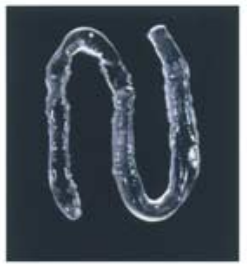

$0.15 \%$ FBS

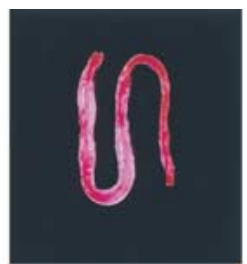

$15 \%$ FBS

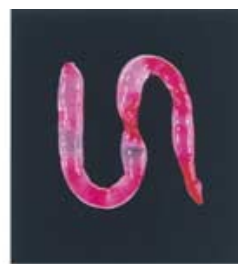

$1.5 \%$ FBS

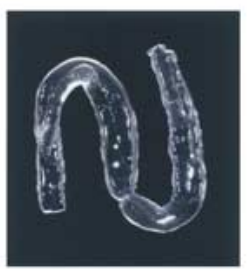

0\% FBS
Fig. 8. Calcification of rat tail tendon after incubation in DMEM containing $2 \mathrm{mM}$ phosphate and the indicated amounts of FBS or in FBS alone. Tail tendons from 40-dayold rats were extracted for 24 hours with 0.5 M EDTA pH 7.5, rinsed thoroughly with water, and dried. Three milligram samples of dry tail tendon were rehydrated in water and placed in $10 \mathrm{ml}$ DMEM containing $2 \mathrm{mM}$ phosphate and the indicated amounts of FBS or in $10 \mathrm{ml}$ FBS alone and were incubated for 6 days in a humidified incubator at $37^{\circ} \mathrm{C}$ and $5 \%$ $\mathrm{CO}_{2}$. After incubation, tendons were stained for calcification with Alizarin red (see online figure for color image). Representative tendon portions are shown for each condition.

$15 \%$ FBS $(1.43 \pm 0.08)$, and $1.5 \%$ FBS $(1.39 \pm 0.04)$ are not significantly different than those of normal tibias $(1.37 \pm 0.03)$, with $P$ values of $0.21,0.26$, and 0.6 , respectively. Examination of von Kossa-stained sections showed that the pattern of calcification in tendon is discontinuous, with numerous discrete calcification foci (Fig. 10).

We employed FTIR spectroscopy and powder X-ray diffraction to compare the mineral phase that accumulates in tendon during incubation in serum containing $15 \%$ FBS to the mineral found in rat tibia. These studies were carried out on samples that had been first deproteinized in order to better focus on the mineral phase of each tissue. Figure 11 shows the FTIR absorbance spectrum of normal tibias and of tendons that have been mineralized by incubation in DMEM containing $15 \%$ FBS and $2 \mathrm{mM} \mathrm{Pi}$. Note the nearly identical spectra, with a $\mathrm{P}-\mathrm{O}$ stretch vibration absorbance peak at around $1040 \mathrm{~cm}^{-1}$ and $\mathrm{P}-\mathrm{O}$ bend vibration absorbance peaks at around 604 and $566 \mathrm{~cm}^{-1}$, as well as a carbonate $\mathrm{C}-\mathrm{O}$ bend vibration absorbance peak at about $874 \mathrm{~cm}^{-1}$ and $\mathrm{C}-\mathrm{O}$ stretch vibration absorbance from 1400 to $1480 \mathrm{~cm}^{-1}$ in each spectrum. Powder X-ray diffraction analysis shows that nondemineralized tibias and tendons mineralized in $15 \%$ FBS in DMEM (Fig. 11 insets) both contain a major diffraction peak at $32 \mathrm{deg}$ and minor peaks at 26 and $50 \mathrm{deg}$, all characteristic of bone apatite.

\section{Discussion}

The initial goal of the present study was to develop a cell-free in vitro model in order to study bone mineralization and its inhibitors. EDTA-demineralized whole tibias were used as a model matrix in these studies so that the location of any in vitro mineralization observed could be compared directly with the location of mineral found in the original tibia prior to demineralization. In the first experiment, demineralized tibias were incubated 6 days at $37^{\circ} \mathrm{C}$ in the absence of any living cells and in a cell culture medium that is similar in many ways to that often used in studies of in vitro mineralization, namely, DMEM containing $15 \%$ fetal bovine serum (FBS) and $10 \mathrm{mM} \beta$-glycerophosphate. Massive remineralization of the demineralized tibias was observed, and the total calcium and phosphate content of the remineralized tibias approached the level found in a nondemineralized control tibia (Fig. 2). The pattern of Alizarin staining for mineralization, however, revealed that mineral deposition was not localized to the diaphysis, as was the case for the original tibia; instead, comparable mineralization was detected in the cartilaginous ends of the bone as well as in the normally mineralized collagen matrix of the midshaft (Fig. 1). A likely explanation for this result is that the efficient hydrolysis of $\beta$-glycerophosphate by the phosphatases present in FBS resulted in a phosphate concentration of nearly $5 \mathrm{mM}$ after 6 days in culture (Fig. 3), and this supraphysiological level of ionic phosphate caused the nonspecific precipitation of mineral within both the cartilage and collagen matrices of the tibia. A similar nonspecific precipitation of mineral has been seen by other investigators using medium containing $10 \mathrm{mM} \beta$-glycerophosphate, and in each instance the mineral precipitation has been associated with the hydrolysis of the $\beta$-glycerophosphate by phosphatases and the associated massive increase in medium levels of phosphate [14-17].

The addition of inorganic phosphate was tested as an alternative method of phosphate supplementation. In an earlier study, Chung et al. [17] had proposed not exceeding $2 \mathrm{mM} \mathrm{Pi}$ in the culture medium in order to prevent nonphysiological mineral formation. When DMEM supplemented with $15 \%$ FBS and containing $2 \mathrm{mM}$ phosphate was tested, we succeeded in remineralizing a demineralized tibia in all sites where mineral is normally found in a nondemineralized tibia (Fig. 1), sites that include the midshaft bone as well as growth plate cartilage. It should be noted that the resulting total mineral content of the tibia after incubation for 6 days at $37^{\circ} \mathrm{C}$ is lower than that found in a nondemineralized tibia (Fig. 2) and that this may reflect the need for longer incubation times to achieve full remineralization. A 6 day incubation time was chosen as a minimum time necessary to see evidence of mineralization by Alizarin stain. Additionally, the medium phosphate concentra- 


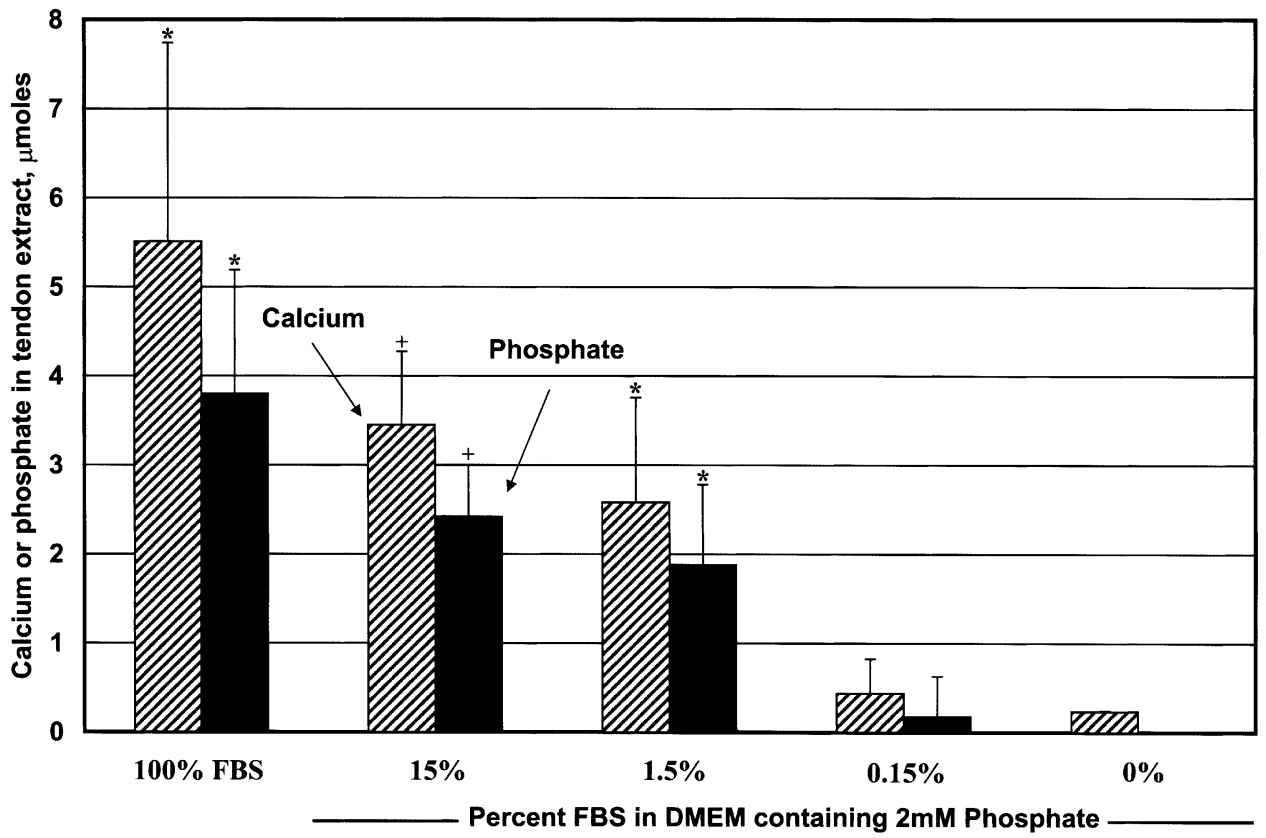

Fig. 9. Calcium and phosphate levels in tendon after incubation for 6 days in DMEM containing $2 \mathrm{mM}$ phosphate and the indicated amounts of FBS or in FBS alone. Rat tail tendons were incubated as described in Figure 8. After incubation, tendons were demineralized in acid and the acid extracts were analyzed for calcium and phosphate as described in Materials and Methods. Each condition was reproduced 4 times. The error bars represent one standard deviation above the mean. Values indicated $(*)$ are significantly different from 0\% FBS $(P<0.01)$. Values indicated $(+)$ are significantly different from 0\% FBS $(P<0.001)$.

tion of $2 \mathrm{mM}$ used to remineralize the tibia is below the concentration where spontaneous precipitation can be seen within the medium after 6 days in culture at $37^{\circ} \mathrm{C}$ in the absence of serum. Therefore, a major finding of these studies is that the direct addition of a known and controlled amount of inorganic phosphate to the incubation medium is preferable to incubation models using $\beta$-glycerophosphate and a source of alkaline phosphatase because in that circumstance a supraphysiological level of phosphate can be generated which has the tendency to result in nonspecific mineral precipitation within the cartilage and bone of the demineralized tibias, rather than the specific remineralization of those regions of bone matrix that were calcified in the original bone.

It was shown previously by Khouja et at. [14], and was confirmed in experiments presented here (Fig. 3), that serum hydrolyzes $\beta$-glycerophosphate to inorganic phosphate and glycerol. When the direct addition of phosphate was used instead of $\beta$-glycerophosphate hydrolysis as a source of phosphate, we theorized that serum would no longer be needed to obtain mineralization, since the alkaline phosphatase that serum contains was no longer required to generate phosphate. Surprisingly, a tibia incubated for 6 day in medium with $\left[\mathrm{PO}_{4}^{3-}\right]=2 \mathrm{mM}$ and $\left[\mathrm{Ca}^{2+}\right]=1.8 \mathrm{mM}$ failed to mineralize in the absence of serum (Fig. 4). However, supplementing the medium with as little as 5\% FBS (and sometimes as little as $1.5 \%$ ) consistently resulted in mineralization of the added tibia and the pattern of mineralization in the tibia was identical to that seen in a nondemineralized tibia (Fig. 4). It is unlikely that this effect can be explained by the impact of $5 \%$ FBS on the ionic milieu of DMEM, since the predicted effect of $5 \%$ serum is to increase medium phosphate by $3 \%$ and to

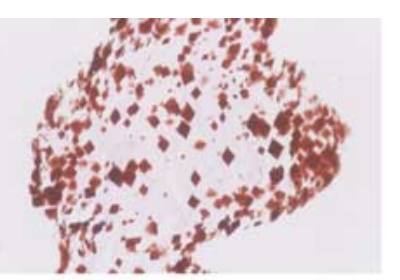

$100 \%$ FBS

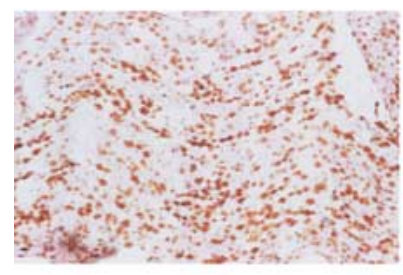

$1.5 \%$ FBS

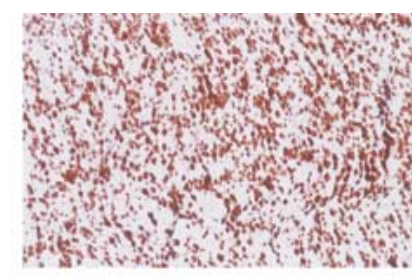

$15 \%$ FBS

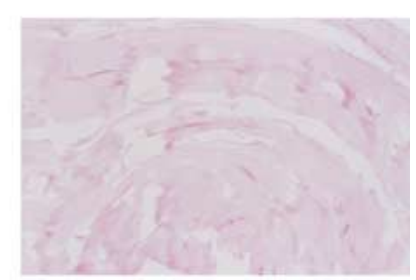

$0.15 \%$ FBS

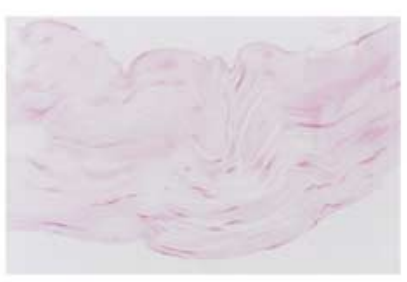

0\% FBS

Fig. 10. Microscopic examination of tendons after incubation for 6 days in DMEM containing $2 \mathrm{mM}$ phosphate and the indicated amounts of FBS or in FBS alone. Rat tail tendons were incubated as described in Figure 8 and then fixed in absolute ethanol for 24 hours. Representative von Kossastained sections are shown for tendons after incubation for 6 days at $37^{\circ} \mathrm{C}$ in DMEM containing $2 \mathrm{mM}$ phosphate and the indicated amounts of FBS or in FBS alone (upper left). von Kossa staining indicates the presence of phosphate mineral. (See online figure for a color image). $400 \times$ magnification. 


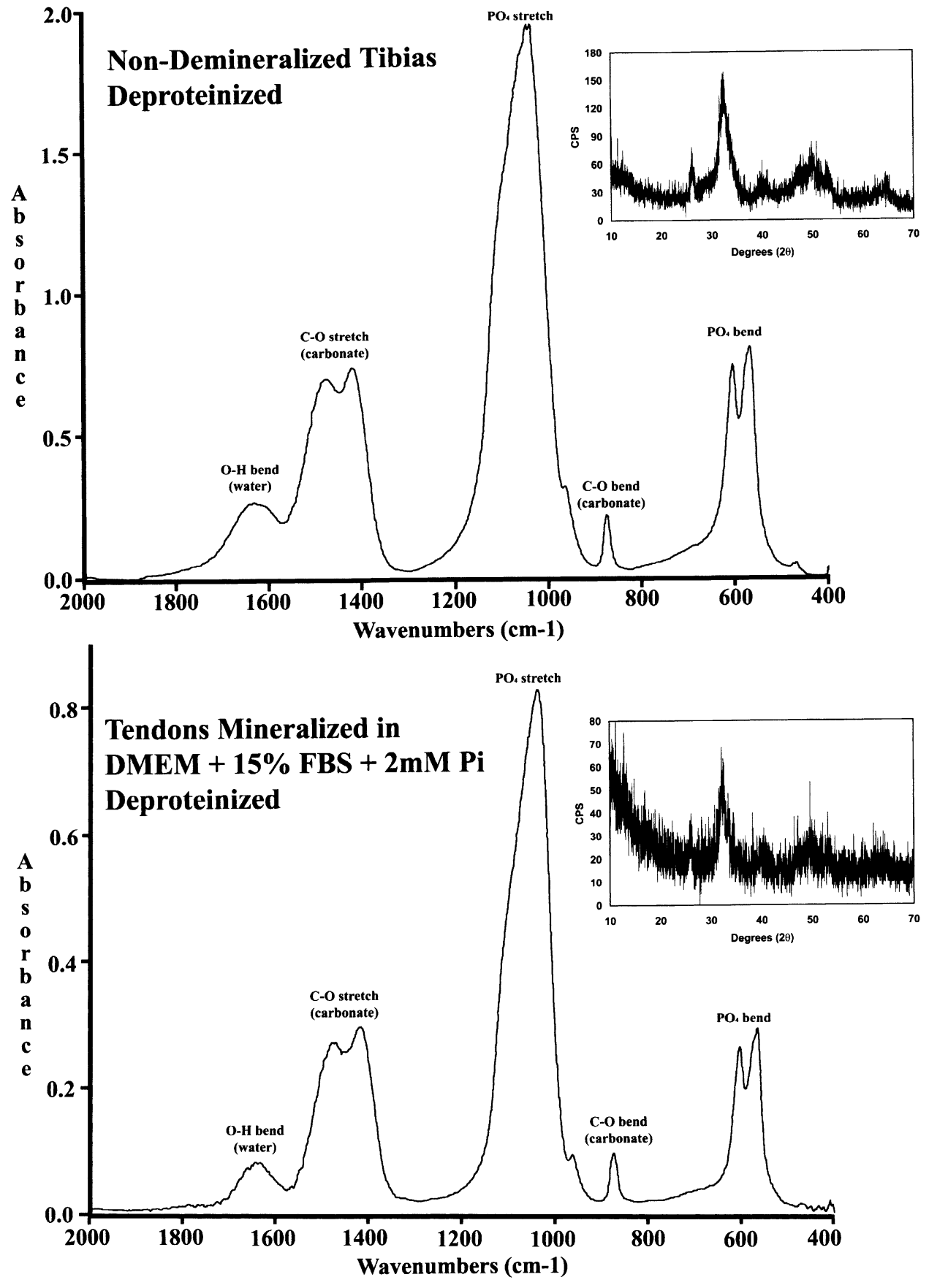

Fig. 11. FTIR spectroscopic and Powder XRD analyses of normal tibias and mineralized tendon. Tendons were EDTAextracted and mineralized in $15 \%$ FBS, then normal tibias and mineralized tendons were prepared for FTIR analysis as described in Materials and Methods. To better compare the mineral phases in normal tibias versus mineralized tendon, tibias and tendon were deproteinized [19]. $\mathrm{KBr}$ transparent pellets were analyzed from 2000 to $400 \mathrm{~cm}^{-1}$ at $4-\mathrm{cm}^{-1}$ resolution for 256 scans. (Top) Newborn rat tibias deproteinized.

(Bottom) Mineralized rat tail tendon deproteinized. Powder $\mathrm{XRD}$ analysis was performed on the same deproteinized samples described in Materials and Methods (insets). decrease medium ionic calcium by $2 \%$. This conclusion is supported by the observation that addition of sufficient calcium and phosphate to DMEM to increase the levels of each by $10 \%$ (to $2 \mathrm{mM}$ calcium and $2.2 \mathrm{mM}$ phosphate) did not cause detectable remineralization of demineralized tibias after incubation for 6 days in the absence of serum (personal observations).

Our data therefore support the hypothesis that there exists in serum one or more nucleators of bone remineralization. Parallel experiments utilizing rat tail tendon in place of tibias as a matrix (Fig. 8-11) suggest that this nucleator(s) functions to mineralize not only a bone matrix but all type I collagen matrices in general. The mineral phase introduced into bone and tendon by incubation in DMEM containing FBS is nearly identical in $\mathrm{Ca} / \mathrm{PO}_{4}$ ratio and in FTIR and powder X-ray diffraction spectra to the mineral found in tibias prior to demineralization (Fig. 7 and 11).

It is not yet clear why a nucleator that initiates the specific mineralization of type I collagen matrices might be found in serum. One possibility is that the serum nucleator actually arises in bone and performs a critical function in normal bone mineralization. Most bone turnover in the adult occurs within the basic multicellular 
unit (BMU), an anatomic structure that contains boneresorbing osteoclasts and bone-forming osteoblasts [21, 22]. The classical BMU structure is found in cortical bone, but there is recent evidence that a very similar structure, the bone-remodeling compartment (BRC), is found on the surfaces of bone trabeculae in cancellous bone $[23,24]$. Both the BMU and the BRC are vascular compartments, with direct connections to the vascular system and evidence exists for blood-derived cells (such as erythrocytes and leukocytes) within the compartment [21-24]. We speculate that nucleator(s) of bone matrix mineralization are secreted by osteoblasts in the BMU, and that due to the vascular nature of these structures, some of the nucleator unavoidably escapes to blood. The escape of the nucleator from the BMU to serum is supported by the appearance in serum of other proteins synthesized in the BMU [e.g., alkaline phosphatase and bone Gla protein (osteocalcin)] and by the massive accumulation of some serum proteins in the extracellular bone matrix (e.g., fetuin, also called $\alpha$-2HS glycoprotein, which is one of the most abundant noncollagenous proteins in rat, human, and bovine bone [25-27]).

If serum indeed contains a nucleator of type I collagen mineralization, one that normally initiates bone mineralization, one might ask why some tissues that are exposed to blood and contain type I collagen, such as tendon, do not normally mineralize. We speculate that the failure of some type I collagen structures to mineralize in vivo is due to the presence of powerful inhibitors of mineralization. Previous studies have shown that serum itself contains high levels of one potent inhibitor of hydroxyapatite formation, fetuin ( $\alpha 2$-HS-glycoprotein) [28-30], and that the calcification of cartilage in vivo is normally prevented by the calcification inhibitory activity of the vitamin K-dependent matrix Gla protein $[31,32]$. It seems likely that type I collagen matrices that do not ordinarily calcify, such as tendon, contain other potent inhibitors of mineralization in vivo.

The results presented here, when combined with the results from earlier studies of other investigators, may have ramifications for studies employing cell culture systems to study mineralization. Earlier work from two different laboratories indicated that the role of cells in culture might simply be to produce a suitable matrix to be mineralized $[14,33]$. Investigators who use $\beta$-glycerophosphate and cells with alkaline phosphatase activity would be advised to measure medium phosphate values so that they can distinguish between mineralization and precipitation of mineral. Alkaline phosphatase exogenously added, produced by cells in culture, or found in serum will cleave $\beta$-glycerophosphate efficiently, and the resulting phosphate values may be so high as to cause nonphysiological precipitation [14-17]. Furthermore, we have found evidence for a nucleator in serum. Therefore, it is at least theoretically possible that cells which produce a collagen matrix may appear to regulate mineralization when, in fact, they have only produced the matrix which is able to mineralize. We suggest that the demineralized rat tibia model we describe here may serve as a control to test for factors produced by potential mineralizing cells in culture. A simple comparison of the conditioned media from cells and the media alone in their abilities to remineralize newborn rat tibias would provide an adequate test for the presence of cell-generated mineralization components.

In conclusion, we have successfully developed a simple and defined cell-free in vitro system to study mineralization and its inhibitors. In the course of this study, we have found evidence that fetal bovine serum contains one or more nucleators of mineralization. The demineralized newborn rat tibia as a model matrix proved an effective model in part because it allows comparisons of the pattern of mineralization obtained under test conditions with that found in a nondemineralized control tibia at both the macroscopic and microscopic levels. Future work will include investigations aimed toward identifying the serum nucleator(s) found in this study.

Acknowledgments. We are grateful to Dr. Murray Goodman (University of California, San Diego) for use of his FTIR spectrometer and to Matthew Williamson for critical review of the manuscript. N. Hamlin is partially supported by a GAANN (Graduate Assistance in Areas of National Need Department of Education) fellowship. This work was supported in part by Grant AR25921 from The National Institutes of Health

\section{References}

1. Ahmad M, McCarthy M, Gronowicz G (1999) An in vitro model for mineralization of human osteoblast-like cells on implant materials. Biomaterials 20:211-220

2. Casser-Bette M, Murray AB, Closs EI, Erfle V, Schmidt J (1990) Bone formation by osteoblast-like cells in a threedimensional cell culture. Calcif Tissue Int 46:46-56

3. Chang Y-L, Stanford CM, Keller JC (2000) Calcium and phosphate supplementation promotes bone cell mineralization: Implications for hydroxyapatite (HA)-enhanced bone formation. J Biomed Mater Res 52:270-278

4. Cheng S-L, Yang JW, Rifas L, Zhang S-F, Avioli LV (1994) Differentiation of human bone marrow osteogenic stromal cell in vitro: Induction of the osteoblast phenotype by dexamethasone. Endocrinology 134:277-286

5. Dean DD, Schwartz Z, Bonewald L, Muniz OE, Morales S, Gomez R, Brooks BP, Qiao M, Howell DS, Boyan BD (1994) Matrix vesicles produced by osteoblast-like cells in culture become significantly enriched in proteoglycandegrading metalloproteinases after addition of $\beta$-glycerophosphate and ascorbic acid. Calcif Tissue Int 54:399-408

6. Ecarot-Charrier B, Glorieux FH, Van Der Rest M, Pereira G (1983) Osteoblasts isolated from mouse calvaria initiate matrix mineralization in culture. J Cell Biol 96:639-643

7. Gerstenfeld LC, Chipman SD, Glowacki J, Lian JB (1987) Expression of differentiated function by mineralizing cultures of chicken osteoblasts. Dev Biol 122:49-60

8. Kato Y, Boskey A, Spevak L, Dallas M, Hori M, Bonewald LF (2001) Establishment of osteoid preosteocyte-like 
cell MLO-A5 that spontaneously mineralizes in culture. $\mathbf{J}$ Bone Miner Res 16:1622-1633

9. Bellows CG, Aubin JE, Heersche JNM, Antosz ME (1986) Mineralized bone nodules formed in vitro from enzymatically released rat calvaria cell populations. Calcif Tissue Int 38:143-154

10. Rey C, Kim H-M, Gerstenfeld L, Glimcher MJ (1995) Structural and chemical characteristics and maturation of the calcium-phosphate crystals formed during the calcification of the organic matrix synthesized by chicken osteoblasts in cell culture. J Bone Miner Res 10:1577-1588

11. Rey C, Kim H-M, Gerstenfeld L, Glimcher MJ (1996) Characterization of the apatite crystals of bone and their maturation in osteoblast cell culture: Comparison with native bone crystals. Connect Tissue Res 35:343-349 [397403]

12. Robey PG, Termine JD (1985) Human bone cells in vitro. Calcif Tissue Int 37:453-460

13. Schoeters G, Leppens H, Van Gorp U, Van Den Heuvel R (1992) Haemopoeitic long-term bone marrow cultures from adult mice show osteogenic capacity in vitro on 3dimensional collagen sponges. Cell Prolif 25:587-603

14. Khouja HI, Bevington A, Kemp GJ, Russell RGG (1990) Calcium and orthophosphate deposits in vitro do not imply osteoblast-mediated mineralization: Mineralization by betaglycerophosphate in the absence of osteoblasts. Bone $11: 385-391$

15. Boskey AL, Guidon P, Doty SB, Stiner D, Leboy P, Binderman I (1996) The mechanism of $\beta$-glycerophosphate action in mineralizing chick limb-bud mesenchymal cell cultures. J Bone Miner Res 11:1694-1702

16. Gronowicz G, Woodiel FN, McCarthy M-B, Raisz LG (1989) In vitro mineralization of fetal rat parietal bones in defined serum-free medium: Effect of $\beta$-Glycerophosphate. J Bone Miner Res 4:313-324

17. Chung C-H, Golub EE, Forbes E, Tokuoka T, Shapiro IM (1992) Mechanism of action of $\beta$-glycerophosphate on bone cell mineralization. Calcif Tissue Int 51:305-311

18. Chen PS, Toribara TY, Warner H (1956) Microdetermination of phosphorus. Anal Chem 28:1756-1758

19. Weiner S, Price PA (1986) Disaggregation of bone into crystals. Calcif Tissue Int 39:365-375

20. Smith BC (1999) Infrared Spectral Interpretation-A Systematic Approach. CRC Press LLC, Boca Raton, FL,

21. Parfitt A (1998) Osteoclast precursors as leukocytes: importance of the area code. Bone 23:491-494
22. Parfitt A (2000) The mechanism of coupling: a role for the vasculature. Bone 26:319-323

23. Parfitt AM (2001) The bone remodeling compartment: a circulatory function for bone lining cells. J Bone Miner Res 16:1583-1585

24. Hauge EM, Qvesel D, Eriksen EF, Mosekilde L, Melsen F (2001) Cancellous bone remodeling occurs in specialized compartments lined by cells expressing osteoblastic markers. J Bone Miner Res 16:1575-1582

25. Ohnishi T, Arakaki N, Nakamura O, Hirono S, Daikuhara Y (1991) Purification, characterization, and studies on biosynthesis of a $59-\mathrm{kDa}$ bone sialic acid-containing protein (BSP) from rat mandible using a monoclonal antibody. J Biol Chem 266:14636-14645

26. Quelch K, Cole W, Melick R (1984) Noncollagenous proteins in normal and pathological human bone. Calcif Tissue Int 36:545-549

27. Ashton BA, Triffitt JT, Herring GM (1974) Isolation and partial characterization of a glycoprotein from bovine cortical bone. Eur J Biochem 45:525-533

28. Price PA, Lim JE (2003) The inhibition of calcium phosphate precipitation by fetuin is accompanied by the formation of a fetuin-mineral complex. J Biol Chem 278:22144-22152

29. Price PA, Thomas GT, Pardini AW, Figueira WF, Caputo J, Williamson MK (2002) Discovery of a high molecular weight complex of calcium, phosphate, fetuin, and matrix Gla protein in the serum of etidronate-treated rats. J Biol Chem 277:3926-3934

30. Schinke T, Amendt C, Trindl A, Poschke O, Muller-Esterl W, Jahnen-Dechent W (1996) The serum protein alpha 2HS glycoprotein/fetuin inhibits apatite formation in vitro and in mineralizing calvaria cells. J Biol Chem 271:2078920796

31. Price PA, Faus SA, Williamson MK (1998) Warfarin causes rapid calcification of the elastic lamellae in rat arteries and heart valves. Arterioscler Thromb Vasc Biol 18:1400-1407

32. Luo G, Ducy P, McKee MD, Pinero GJ, Loyer E, Behringer RR, Karsenty G (1997) Spontaneous calcification of arteries and cartilage in mice lacking matrix Gla protein. Nature 386:78-81

33. Marsh ME, Munne AM, Vogel JJ, Cui Y, Franceschi RT (1995) Mineralization of bone like extracellular matrix in the absence of functional osteoblasts. J Bone Miner Res 10:1635-1643 